\title{
A negative feedback loop involving small RNAs accelerates Vibrio cholerae's transition out of quorum-sensing mode
}

\author{
Sine L. Svenningsen, ${ }^{1}$ Christopher M. Waters, ${ }^{1,2}$ and Bonnie L. Bassler ${ }^{1,2,3}$ \\ ${ }^{1}$ Department of Molecular Biology, Princeton University, Princeton, New Jersey 08544, USA; ${ }^{2}$ Howard Hughes Medical \\ Institute, Department of Molecular Biology, Princeton University, Princeton, New Jersey 08544, USA
}

\begin{abstract}
Quorum sensing is a cell-to-cell communication process that allows bacteria to measure their population numbers and to synchronously alter gene expression in response to changes in cell population density. At the core of the Vibrio cholerae quorum-sensing signal transduction pathway lie four redundant small RNAs (sRNAs), named the Quorum Regulatory RNAs (Qrr1-4). Expression of qrr1-4 is cell population density-dependent due to a requirement for the quorum-sensing controlled phosphorylated response regulator LuxO-P, which is abundant only at low cell population density. When expressed, Qrr1-4 repress translation of HapR, the "master" quorum-sensing transcription factor. Here we show a negative feedback loop in which HapR activates transcription of the qrr genes, which indirectly leads to hapR repression. Efficient feedback activation of the qrr genes requires the simultaneous presence of LuxO-P (present only at low cell population density) and HapR (present only at high cell population density). For this reason, the feedback loop does not influence quorum sensing at steady-state low or high cell population density. However, LuxO-P and HapR are simultaneously present immediately following the switch from high to low cell density conditions. In this state, the HapR feedback loop dramatically accelerates $V$. cholerae's transition from the high to the low cell density mode.
\end{abstract}

[Keywords: Quorum sensing; small RNA; negative feedback loop]

Supplemental material is available at http://www.genesdev.org.

Received May 29, 2007; revised version accepted November 2, 2007.

Bacteria detect and integrate multiple environmental inputs and respond with appropriate behavioral outputs. For many crucial bacterial behaviors, the numbers and composition of the microbial community are key environmental parameters (Miller et al. 2002; Smith and Iglewski 2003). Quorum sensing is a widespread mechanism of cell-cell communication used by both Gramnegative and Gram-positive bacteria to monitor the cell density and diversity of the immediate bacterial population (Henke and Bassler 2004). Quorum-sensing bacteria produce, secrete, and subsequently detect signal molecules called autoinducers (AIs). Where cells are sparse, AIs are secreted but do not accumulate to detectable levels. As cell population density increases, so does the extracellular concentration of AIs. Upon reaching a critical concentration, the AIs are detected by cognate sensor proteins that either transduce the signal to downstream transcriptional regulators, or themselves function as transcriptional regulators, to mediate changes in gene expression (for review, see Waters and Bassler 2005). In

\footnotetext{
${ }^{3}$ Corresponding author.
}

E-MAIL bbassler@princeton.edu; FAX (609) 258-2957.

Article is online at http://www.genesdev.org/cgi/doi/10.1101/gad.1629908. its simplest form, quorum sensing allows bacteria to exist in one of two behavioral modes: an "individual cell mode," appropriate for low cell density conditions, and a "social mode," appropriate for high cell density conditions.

In Vibrio cholerae, the causative agent of the diarrheal disease cholera, quorum sensing controls important processes including virulence factor expression and biofilm formation (Miller et al. 2002; Hammer and Bassler 2003). At least two parallel signaling pathways contribute to $V$. cholerae quorum sensing (Fig. 1; Miller et al. 2002). One pathway is composed of the CqsA-dependent autoinducer CAI-1 and its cognate two-component sensor CqsS. The structure of CAI-1 is unknown, and its synthesis is restricted to $V$. cholerae and closely related $V i b$ rio species, suggesting that it functions in intragenera signaling. The other pathway is composed of the LuxSdependent autoinducer AI-2 and its sensory apparatus LuxPQ. LuxP is a periplasmic protein that binds AI-2 and regulates the activity of the two-component protein LuxQ. AI-2 is the collective name for the family of AIs that are derivatives of 4,5-dihydroxy-2,3-pentanedione (DPD), which interconvert and exist in equilibrium (Schauder et al. 2001; Miller et al. 2004). LuxS and AI-2 
Low Cell Density

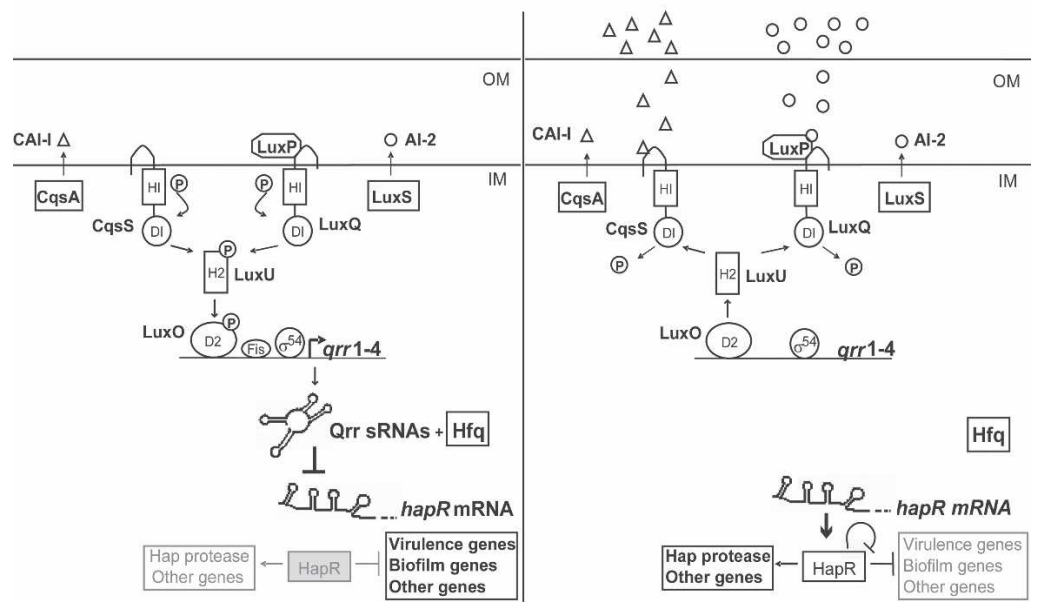

Figure 1. Model of the $V$. cholerae quorumsensing circuit. Two quorum-sensing pathways converge at LuxO to control transcription of four genes encoding the sRNAs Qrrl-4. Qrrl-4, in turn, bind to and repress translation of the hapR mRNA. Details of the signal transduction mechanism are provided in the text. Triangles and circles represent the AIs CAI- 1 and AI-2, respectively. OM and IM indicate outer membrane and inner membrane, respectively. The $\mathrm{P}$ in the circle indicates that the protein is in the phosphorylated state. are widespread in the bacterial kingdom and are proposed to mediate interspecies communication (Xavier and Bassler 2003).

At low cell population density, the two-component proteins CqsS and LuxQ act as kinases to funnel phosphate via LuxU to the response regulator LuxO (Fig. 1, left panel). The phosphorylated form of LuxO (LuxO-P) functions as a transcriptional activator in conjunction with the $\sigma^{54}$ subunit of RNA polymerase. Together, they promote transcription of the qrr 1-4 (Quorum Regulatory RNA) genes encoding four unlinked, homologous small RNAs (sRNAs). Transcription of qrr $1-4$ is enhanced in the presence of the nucleoid protein Fis, whose expression peaks at low cell population density (Lenz and Bassler 2007). With the assistance of the RNA chaperone Hfq, Qrr1-4 base-pair with the 5' untranslated region (UTR) of the hapR mRNA and promote its degradation (Lenz et al. 2004). Thus, when Qrr1-4 are expressed, HapR synthesis is repressed. HapR is the "master" transcriptional regulator of quorum sensing, so in its absence quorum-sensing-repressed genes are expressed and quorum-sensing-activated genes are not.

At high cell population density-i.e., when the AIs bind their cognate two-component sensors-the sensors act as phosphatases, reversing the phosphate flow through the quorum-sensing circuit. This results in dephosphorylation and inactivation of LuxO (Fig. 1, right panel). As a consequence, expression of qrr1-4 is terminated, HapR is produced and regulates its target genes (Lenz et al. 2004). One such target gene is hapR itself, which is autorepressed at high cell population densities (Lin et al. 2005). HapR is a member of the TetR family of DNA-binding regulators. Generally, these proteins function as repressors (Pan and Spratt 1994). HapR is also known to be an activator of gene expression; however, the molecular mechanism underlying activation is not defined (Miyamoto et al. 1994; Jobling and Holmes 1997). Recently, environmental and metabolic inputs other than the AI signals have been shown to channel additional information into the $V$. cholerae quorum- sensing pathway (Lenz et al. 2005; Lenz and Bassler 2007).

Here we investigate the regulatory relationship between the Qrr sRNAs and HapR. We find that HapR can activate transcription of the qrr genes, creating a negative feedback loop in the quorum-sensing circuit. Thus, HapR both directly and indirectly represses its own production. Direct repression of the hapR promoter by HapR only occurs at high cell density, and prevents overproduction of HapR in the social mode (Lin et al. 2005). In contrast, the post-transcriptional repression of hapR, via the Qrr sRNA feedback loop, requires the presence of both LuxO-P and HapR, and therefore only occurs at the transition from high to low cell density conditions. This latter feedback loop dramatically accelerates the transition of $V$. cholerae cells out of social mode and into individual cell mode.

\section{Results}

\section{hapR mRNA and Qrr sRNA levels vary reciprocally}

Previous studies in $V$. cholerae suggested that the Qrr sRNAs are expressed at low cell density, when the transcriptional activator LuxO is phosphorylated, but not at high cell density when LuxO is dephosphorylated and inactive (Lenz et al. 2004). A reciprocal pattern for hapR mRNA was inferred; i.e., it was expected to be degraded at low cell density when the Qrr sRNAs are present, and abundant at high cell density when the Qrr sRNAs are absent (Fig. 1). We tested these predictions by measuring hapR mRNA and Qrr1-4 sRNA levels during growth using Northern blotting (Fig. 2A). As anticipated, the four Qrr sRNAs were abundant at low cell density and very little Qrr sRNA could be detected at high cell density. In contrast, only low levels of hapR mRNA were present at low cell density, while high levels of hapR mRNA accumulated at high cell density. This reciprocal relationship between Qrr sRNA levels and hapR mRNA levels supports a model in which cell density-dependent gene regu- 
Svenningsen et al.

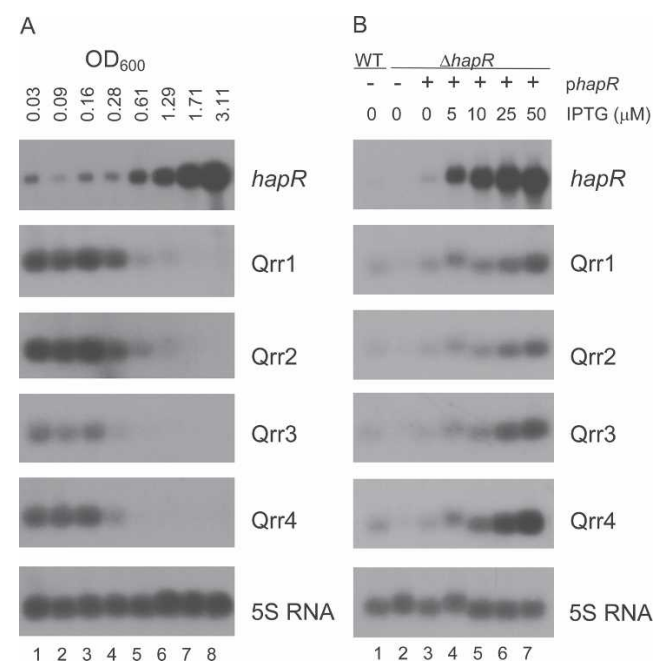

Figure 2. hapR mRNA and Qrr sRNA patterns of accumulation. (A) An overnight wild-type $V$. cholerae culture was diluted 1:1000 into fresh LB medium. Total RNA was collected at various times during growth $\left(\mathrm{OD}_{600}\right)$. (B) Overnight cultures of $V$. cholerae strains-wild type (lane 1), $\Delta$ hapR (lane 2), or a $\Delta$ hapR mutant expressing hapR from pSLS13 (lanes 3-7) -were diluted into fresh LB medium containing the indicated IPTG concentrations and grown to $\mathrm{OD}_{600}=0.1$, at which point total RNA was collected. In both panels, the RNA species indicated on the right were visualized by Northern blotting. 5S RNA is shown as a loading control. The experiment was repeated twice with similar results.

lation in $V$. cholerae is accomplished by the sRNA-mediated degradation of hapR mRNA at low cell density, but not at high cell density (Fig. 1; Lenz et al. 2004).

\section{hapR mRNA levels influence Qrr1-4 levels}

Hfq-dependent sRNAs like Qrr1-4 function by promoting the coupled degradation of the sRNA and its target mRNA upon pairing (Masse et al. 2003). Thus, target mRNA levels can affect the levels of the sRNA partner. We assayed whether deliberately altering the levels of hapR mRNA had any consequence on the Qrr sRNAs. We compared the levels of Qrr1-4 at low cell density in wild-type $V$. cholerae with those in an otherwise isogenic $\Delta$ hapR mutant (Fig. 2B, cf. lanes 1 and 2). Surprisingly, although modest, the abundance of all four Qrr sRNAs decreased in the absence of hapR. This parallel effect of hapR on Qrr sRNA levels was contrary to our expectations based on the data in Figure 2A, where a cell density-dependent increase in hapR mRNA was accompanied by a decrease in Qrr sRNA. We constructed a plasmid capable of inducible expression of full-length hapR mRNA to further manipulate the hapR mRNA:Qrr sRNA ratio. Increased hapR mRNA levels at low cell density resulted in increased Qrr levels (Fig. 2B, lanes 3-7). Together, these results indicate that although the Qrr sRNAs and their target hapR mRNA accumulate reciprocally over the growth curve, increased hapR expression causes an increase in Qrr sRNAs.
HapR is a transcriptional activator of the qrr1-4 promoters

The hapR-induced increase in Qrr sRNAs could be a consequence of increased transcription of the qrr genes, increased stability of the Qrr sRNAs, or both. We first tested whether HapR could activate transcription of qrr 1-4. To do this, we constructed lux reporter fusions to the +1 transcriptional start sites of the four qrr promoters, and measured their expression in wild-type and $\Delta$ hapR mutant $V$. cholerae strains. Figure 3 shows that, in the wild type, each qrr gene is expressed in a cell density-dependent manner (Fig. 3, closed triangles) and, in all cases, deletion of hapR causes a roughly fivefold reduction in qrr-lux expression (Fig. 3, open squares). Thus, transcription of all four qrr genes is indeed sensitive to HapR. When we supplied additional copies of $h a p R$ on a plasmid, transcription of the qrr genes in-

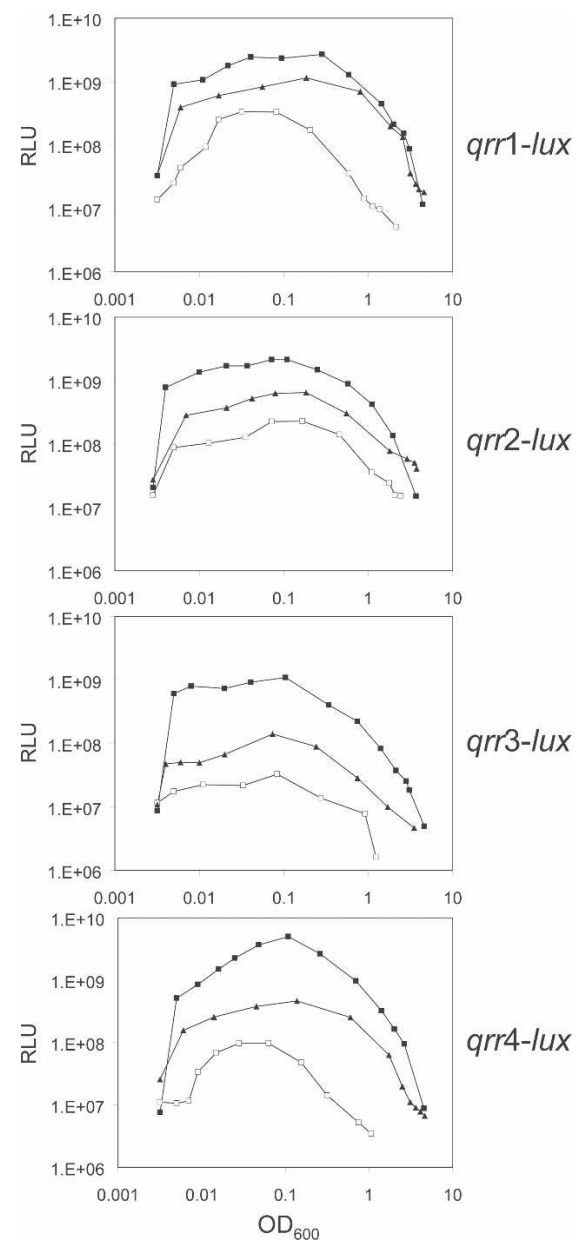

Figure 3. HapR activates transcription of the qrr promoters. Light production from the indicated qrr-lux constructs was measured in $V$. cholerae wild type (closed triangles), a $\Delta$ hapR mutant (open squares), and a $\Delta$ hapR mutant constitutively expressing hapR from pSLS13 (closed squares) during growth following 1:1000 dilution of a dense overnight culture. (RLU) Relative light units. The experiment was repeated on three different occasions with similar results. 
creased up to 50 -fold over the levels in the $\Delta$ hapR mutant (Fig. 3, closed squares).

The simplest explanation for the above results is that the HapR protein activates transcription of the qrr genes. However, since we know that hapR mRNA interacts with the Qrr sRNAs, we tested whether HapR protein is in fact necessary and sufficient for activation of $q r$ rranscription. To do this, we measured light production from the qrr-lux promoter fusions in a $\Delta$ hapR mutant strain following induction of the hapR gene complete with its 5' UTR (Fig. 4A, closed circles), the hapR coding region lacking the 5' UTR (Fig. 4A, closed squares), or a hapR allele containing a missense mutation that does not affect transcription of hapR mRNA but renders HapR incapable of DNA binding and gene activation (Fig. 4A, closed triangles; Hammer and Bassler 2003). Results for the qrr4-lux promoter fusion are shown in Figure 4A; similar results were obtained for qrr1-3. Activation of transcription of qrr4-lux occurs only in the presence of functional HapR protein (Fig. 4A, cf. closed circles and closed triangles). This activation is irrespective of the presence or absence of the $5^{\prime}$ UTR (Fig. 4A, cf. closed

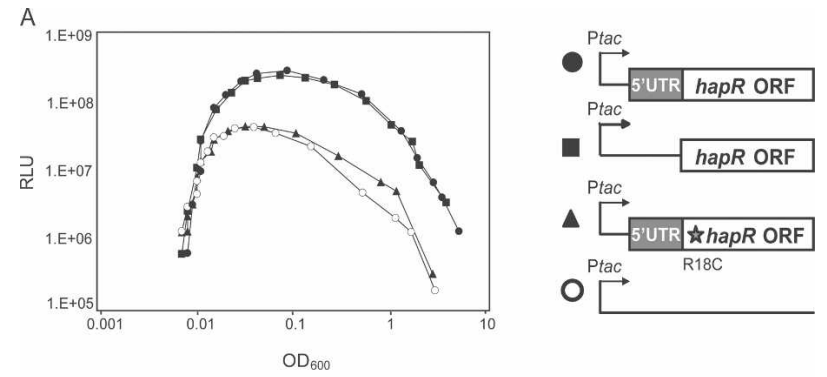

B

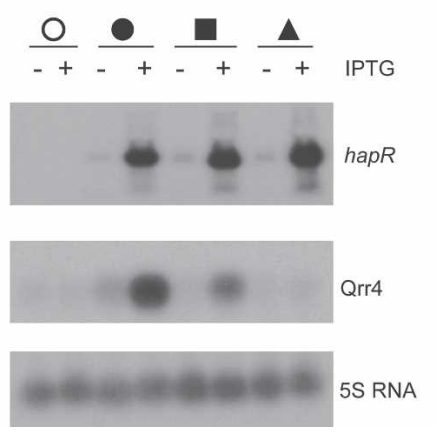

Figure 4. HapR protein is both necessary and sufficient for activating transcription of the qrr4 gene. (A) Light production from the qrr4-lux promoter fusion was measured in the V.cholerae $\Delta$ hapR mutant expressing full-length hapR mRNA (pSLS13, closed circles), the hapR ORF lacking the 5' UTR (pSLS28, closed squares), full-length hapR mRNA producing inactive HapR protein (pSLS30, closed triangles), or the vector alone (pFED342, open circles) during growth $\left(\mathrm{OD}_{600}\right)$ following 1:1000 dilution of a dense overnight culture. (RLU) Relative light units. The experiment was repeated twice with similar results. $(B)$ Accumulation of hapR mRNA and Qrr4 sRNA in a V. cholerae $\Delta$ hapR mutant with and without induction of expression from the four plasmids described in $A$ was visualized by Northern blotting. 5S RNA is shown as a loading control. The experiment was repeated twice with similar results. circles and closed squares). This finding demonstrates that it is the HapR protein itself that is crucial for the feedback on qrr expression, and this activity does not involve base-pairing of the hapR mRNA with the sRNAs. We confirmed that the observed increase in Qrr4 sRNA levels occurred only following induction of plasmids producing functional HapR protein (Fig. 4B) by measuring Qrr4 levels with and without induction of the identical hapR constructs described in Figure 4A. Although hapR was transcribed to identical levels from all the plasmids, Qrr4 only accumulated following induction of plasmids expressing functional HapR (Fig. 4B, closed circle and closed square above the blot), as no Qrr4 accumulated following induction of the plasmid carrying hapR harboring a missense mutation (Fig. 4B, closed triangle). Note that expression of hapR lacking its $5^{\prime}$ UTR required the introduction of a nonnative RBS upstream of hapR. Translation from this RBS is less efficient than translation from the natural RBS; hence, less HapR protein is produced, although the gene is transcribed at the same level as hapR carrying its native RBS (Fig. 4B; data not shown). This accounts for why less Qrr4 accumulated following induction of hapR lacking its native $5^{\prime}$ UTR than following induction of intact hapR mRNA.

The finding that Qrrs accumulate only following induction of intact HapR protein suggests that the HapRdependent accumulation of the Qrr sRNAs we observed in Figure 3 is due entirely to transcriptional activation of the qrr promoters, and not due to interactions between the Qrr sRNAs and the hapR mRNA. To verify this assumption, we repeated the experiment shown in Figure 4 in a $\Delta q r r 1-4, \Delta h a p R$ mutant strain. Indeed, induction of the plasmid-borne hapR construct encoding functional HapR promoted identical levels of transcriptional activation of the qrr4-lux reporter fusion in the $\Delta$ qrr 1-4 and the $q r r 1-4^{+}$strains (data not shown). Thus, we conclude that the Qrr sRNAs are not required for activation of qrr transcription.

\section{HapR activation of the qrr promoters appears to be indirect}

To explore the mechanism underpinning HapR activation of qrr transcription, we performed electrophoretic mobility shift assays (EMSA) with purified HapR protein and the $\sim 500$-base-pair (bp) regions upstream of each qrr gene (Fig. 5A). We could not detect binding of HapR to any of the qrr promoters, suggesting that HapR affects qrr expression indirectly. We are certain our purified HapR protein is active because it readily binds the $a p h A$ promoter, a target known to be directly controlled by HapR (Fig. 5A; Kovacikova and Skorupski 2002). Given that HapR is a transcription factor and that we established that HapR controls transcription of the qrr genes, this result was unanticipated. We therefore measured whether HapR could activate qrr expression in recombinant Escherichia coli carrying $V$. cholerae luxO D47E on the chromosome. LuxO D47E is an allele of LuxO that constitutively mimics LuxO-P (Freeman and Bassler 
A

Figure 5. HapR-mediated feedback requires little sequence specificity. (A) EMSAs were performed on the four qrr promoters with purified HapR protein. The $a p h A$ promoter was used as a positive control for HapR binding (aphA shift), and a region downstream from the HapR-binding sites in the $a p h A$ promoter was used as a negative control (aphA nonshift). (B) The minimal region of the qrr 4 promoter required for regulation by LuxO-P, $\sigma^{54}$, and HapR. Green shading indicates the LuxO-P-binding sites, light blue indicates the 90-nt region between the LuxO-P- and $\sigma^{54}$-binding sites, and dark blue indicates the $\sigma^{54}$-binding site. $(C)$ Light production from the indicated reporter constructs in a $V$. cholerae $\Delta$ hapR mutant expressing hapR from pSLS13 (black bars) or carrying the vector alone (white bars) induced with $50 \mu \mathrm{M}$ IPTG. The experiment was repeated three times, each time in triplicate; error bars indicate one standard deviation from the mean. Purple shading indicates the $\sigma^{54}$-binding region of the fla $\mathrm{A}$ promoter and pink shading indicates the FlrC-binding region in the flaA promoter. $(D)$ Overnight cultures of $V$. cholerae strains- $\Delta q r r 1-3$ (first lane), $\Delta q r r 1-3, \Delta$ hapR (second lane), $\Delta$ qrr 1-3, Pqrr4 ${ }^{\text {scr }}$ (third lane), or $\Delta q r r 1-3$, $\mathrm{P} q r r^{\mathrm{scr}}$, $\Delta$ hapR (fourth lane)-were diluted 1:1000 into fresh LB medium and grown to $\mathrm{OD}_{600}=0.1$. The RNA species indicated on the right were visualized by Northern blotting. 5S RNA is shown as a loading control. The experiment was repeated twice.

B

C

D
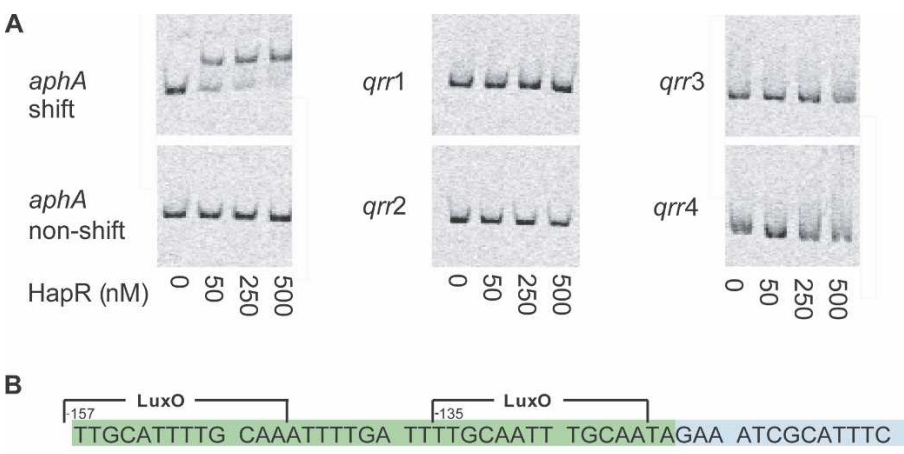
GTCATTCGTT TATGAGTGTG GTTGTAATGA AACTGTTCGT TTTTTGCCCG ATTTTCATGC AAAAGTCGCT AATGAAAAGT TGGCACGTAA TCTGCATTTA ${ }^{-7}$ TTAGATTG $^{+1}$ luXCDABE
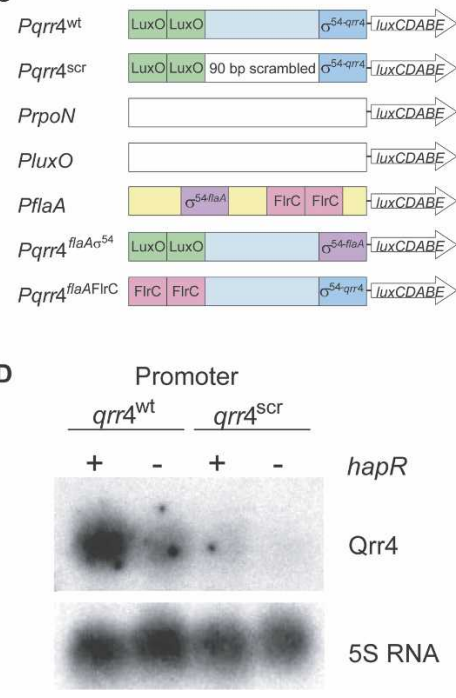

1999|. In E. coli, LuxO D47E activated all four $q r r-g f p$ promoter fusions, but importantly, no increase in transcription occurred in any case following introduction of $h a p R$. These results suggest that HapR feedback is indirect, requiring one or more unidentified mediators that must be present in $V$. cholerae but absent or unresponsive to HapR in E. coli.

\section{HapR-mediated activation of the qrr promoters requires little sequence specificity}

Usually, accessory factors that enhance activation of $\sigma^{54}$-promoters facilitate the interaction of the LuxO-Ptype response regulator with $\sigma^{54}$ by bending the DNA between their respective binding sites, thereby promoting DNA loop formation (Huo et al. 2006). Fis is one such DNA-bending protein that is known to bind the qrr promoters and enhance their transcription (Lenz and Bassler 2007). To test whether HapR feedback is mediated by Fis, we measured the effect of HapR overexpression on qrr4 transcription in the wild type, and compared this effect with that in a $\Delta$ fis mutant. Identical levels of
HapR-dependent qrr4-lux activation occurred in the wild type and in the $\Delta$ fis mutant (data not shown). Thus, we conclude that HapR feedback does not act through Fis.

The shortest region of the qrr4 promoter required for activation by LuxO-P, $\sigma^{54}$, and HapR is shown in Figure $5 \mathrm{~B}$. lux expression from this minimal promoter is shown in Figure 5C $\left(\right.$ Pqrr $\left.^{\mathrm{wt}}\right)$ in the presence of hapR overexpression (black bar) and in the absence of hapR (white bar). In an attempt to pinpoint the region of the qrr4 promoter required for the HapR feedback, the 90-bp region between the LuxO-P-and $\sigma^{54}$-binding sites was mutated (Fig. 5B, light blue) by systematically randomizing and replacing every 15 -bp segment of this region. None of these mutations affected the HapR feedback control of qrr4 (data not shown). We also scrambled the entire 90nucleotide region between the LuxO-P- and $\sigma^{54}$-binding sites by engineering promoters with appropriate spacing but containing only the LuxO-P- and $\sigma^{54}$-binding sites intact. These promoters exhibited reduced transcription compared with the wild-type promoter, but surprisingly, remained responsive to regulation by HapR (one representative is shown in Fig. 5C, Pqrr $4^{\text {scr }}$. To verify this result in its natural context we replaced the wild-type 
chromosomal copy of the qrr4 promoter with the engineered $q r r 4^{\text {scr }}$ promoter construct. We engineered this change into a $\Delta q r r 1-3$ strain that was either hap $R^{+}$or $h a p R^{-}$. Figure 5D shows that the chromosomal $q r r 4^{\text {scr }}$ promoter, while expressed to a lower absolute level than the wild-type qrr 4 promoter, is nonetheless equally responsive as the wild-type promoter to physiological levels of hapR. Therefore, we conclude that the specific sequence between the LuxO-P-and $\sigma^{54}$-binding sites is not required for HapR-mediated control of qrr expression.

Possible explanations for our results are that HapR regulates luxO or rpoN (encoding $\sigma^{54}$ ) expression, HapR affects the activity of the LuxO-P or $\sigma^{54}$ proteins, or alternately, the putative factor required for feedback binds at a site overlapping the LuxO-P- or $\sigma^{54}$-binding sites in the qrr promoters. To test the first possibility, we engineered transcriptional lux fusions to the luxO and $r p o N$ promoters. Neither promoter is regulated by HapR, excluding this possibility (Fig. 5C). To address whether a site overlapping the $\sigma^{54}$-binding site is required, we replaced the $\sigma^{54}$ site of $q$ rr 4 with the corresponding $\sigma^{54}$. binding site from flaA, a hapR-independent $\sigma^{54}$-regulated promoter in $V$. cholerae (Fig. 5C, PflaA). Exchanging the $\sigma^{54}$-binding sites $(-30$ to +1$)$ of $q r r 4$ with that of flaA did not affect feedback by HapR, suggesting that the HapR feedback does not act through DNA sequences overlapping the $\sigma^{54}$-binding site (Fig. 5C, Pqrr $4^{\text {flaAo54 }}$ ). Also, because the fla $A$ promoter as well as two other $\sigma^{54}$ promoters tested (flr $B$ and $g \ln A p 2$ ) (data not shown) are not regulated by HapR, HapR cannot generically influence the activity of $\sigma^{54}$. To test whether the LuxO-Pbinding site is critical for HapR feedback, we also exchanged that site. The flaA promoter is activated by a LuxO-type response regulator called FlrC. Replacing the LuxO-binding sites (-157 to -123$)$ of qrr4 with the analogous FlrC-binding sites from flaA, put qrr4 under FlrC control, but did not affect HapR-mediated feedback (Fig. 5C, Pqrr $4^{f l a A F l r C}$ ). This finding suggests that HapR does not require DNA sequences overlapping the LuxO-binding sites for feedback. Again, using similar logic to that above, since HapR can control qrr4 in conjunction with the two-component protein $\mathrm{FlrC}$, it cannot be the case that HapR control over LuxO activity is responsible for the feedback we observe at the qrr promoters.

Our experiments did not precisely define the region required by HapR for feedback on qrr expression. However, we were able to show that HapR-mediated feedback on the qrr promoters does not act through any of the three known qrr-promoter-binding proteins; LuxO-P, $\sigma^{54}$, or Fis. Furthermore, we suspect that feedback requires binding of an additional factor at the qrr promoters because HapR itself does not appear to fulfill this function. If so, this factor must bind DNA in a relatively sequence-independent manner because we replaced every nucleotide in the qrr 4 promoter without successfully disrupting the feedback. Several transcriptional regulators such as HU (Swinger and Rice 2004) and H-NS (Rimsky 2004) bind DNA in a sequence-independent fashion because, rather than recognizing DNA sequence per se, such factors recognize topological features of the DNA such as intrinsic bends and other structural characteristics.

We tested whether HU mediates HapR feedback on the qrr genes by deleting hupA and hupB, the two genes encoding HU in $V$. cholerae. Identical levels of HapRdependent qrr4-lux activation occurred in wild type, the hupA and hupB single mutants, and the hupA, hupB double mutant (data not shown). Thus, we conclude that HU is not involved in HapR feedback on the qrr genes. We were unable to construct an hns mutant strain. To our knowledge, deletion of hns in V. cholerae El Tor has not been reported, and we suggest that $h n s$ is essential in $V$. cholerae El Tor, although we note that $V$. cholerae Classical hns-null mutations have been obtained (Nye et al. 2000; Tendeng et al. 2000; Krishnan et al. 2004). To circumvent this problem, we examined the hns promoter sequence for putative HapR-binding sites (based on the consensus sequence reported by Lin et al. [2005]). We identified two putative HapR-binding sites, and using EMSA, we confirmed that purified HapR protein binds the hns promoter in vitro. In addition, HapR activates an hns-lux promoter fusion in vivo (data not shown). Consistent with this result, we observed that in E. coli carrying luxO D47E, qrr4-gfp expression is fivefold higher in the wild type than in the corresponding $E$. coli hns mutant (data not shown). Based on these results, we hypothesized that HapR could activate qrr transcription indirectly by activating hns expression. H-NS, in turn, could activate qrr transcription. This mechanism predicts that HapR-dependent activation of the qrr genes would be eliminated following mutation of the HapRbinding sites in the hns promoter. To test this prediction, we constructed a strain lacking the HapR-binding sites at the chromosomal hns promoter. Using EMSA, we verified that HapR does not bind the mutated hns promoter in vitro (data not shown). We found that HapRdependent qrr4-lux activation was identical in the HapRbinding site mutant strain and the wild-type strain. Thus, although hns expression is indeed regulated by HapR, H-NS is not the factor required for HapR feedback on the qrr genes. We conclude that HapR-feedback on the qrr genes is indirect, and most likely mediated by a factor that recognizes the qrr promoters in a sequenceindependent manner. This factor is not Fis, HU, or H-NS. We have initiated a genetic screen to identify this putative regulatory component.

\section{HapR feedback on the qrr genes requires the simultaneous presence of HapR and LuxO-P}

It is intriguing to consider when the HapR feedback loop on the $q r r$ genes would operate. $\sigma^{54}$-RNA polymerase holoenzymes (RNAPs), unlike $\sigma^{70}$-RNAPs, require the ATPase activity of a cognate two-component responseregulator protein, like LuxO-P, to fuel the transition from closed to open complex formation at the initiation of transcription (Morett and Buck 1989; Popham et al. 1989). In all cases studied, the phosphorylated response 
regulators are essential for any transcription to occur from the corresponding $\sigma^{54}$-promoters (Popham et al. 1989; Wedel and Kustu 1995; Buck et al. 2000). Thus, presumably LuxO-P is required for HapR-feedback activation of the qrr promoters. The conundrum is that, at low cell population density, LuxO-P is abundant, but hapR expression is repressed so little or no HapR is present. In contrast, at high cell population density HapR accumulates, but because phosphorylation of LuxO occurs only in the absence of AIs, there is little if any LuxO-P present. Because of this puzzling situation we examined the requirements for and function of the HapR-mediated feedback loop.

First, we address the situation at steady-state low cell density. Under this condition, all HapR protein that accumulated in the preceding high cell density state should have been degraded or diluted out. Thus, the only HapR present is that produced de novo despite Qrr sRNA repression. To investigate if this level of HapR is sufficient for feedback activation of the qrr promoters, we compared transcription of qrr4-lux in the wild-type and $\Delta$ hapR mutant strains at steady-state low cell density. Dense overnight cultures were diluted to extremely low cell density (1:50,000,000 dilution) and subsequently allowed to grow for $\sim 20$ generations. This extended growth at low cell density was carried out to ensure complete elimination of pre-existing HapR prior to measuring qrr4-lux transcription $\left(\mathrm{OD}_{600}=0.03\right)$. Bioluminescence levels from the qrr4-lux reporter were identical in the wild type and the $\Delta$ hapR mutant (Fig. 6A), suggesting that HapR accumulation at steady-state low cell density is insufficient for feedback activation of the qrr4 promoter. (We note that the HapR-mediated activation of qrr1-4 transcription shown in Fig. 3 was monitored after only a 1:1000 dilution of a dense overnight culture. Presumably, pre-existing HapR is not fully eliminated following this treatment, which accounts for why HapRmediated qrr activation occurs at "low cell density" in the experiments in Fig. 3, but not in the one presented in Fig. 6A.)

Second, we address the situation at steady-state high cell density. Under this condition, HapR is abundant, but LuxO is presumably fully dephosphorylated, and therefore inactive. This time, we examined transcription from the qrr4 promoter in the V. cholerae wild type and the $\Delta$ hapR mutant at very high cell density $\left(\mathrm{OD}_{600}=4\right)$. This extremely high cell density was chosen to ensure that LuxO-P had been reduced to its minimal level, thereby negating any possible "molecular memory" of the preceding low cell density state. Figure 6B shows that HapR noticeably promotes the expression of the qrr4 gene at very high cell density conditions, but relative to $V$. cholerae strains at steady-state low cell density (Fig. 6A), total qrr4-lux transcription is reduced $>100$ fold. This result shows that the HapR feedback loop is negligible under steady-state high cell density conditions.

In summary, these results show that HapR feedback on the qrr promoters indeed requires the simultaneous presence of LuxO-P and HapR. Therefore, the feedback

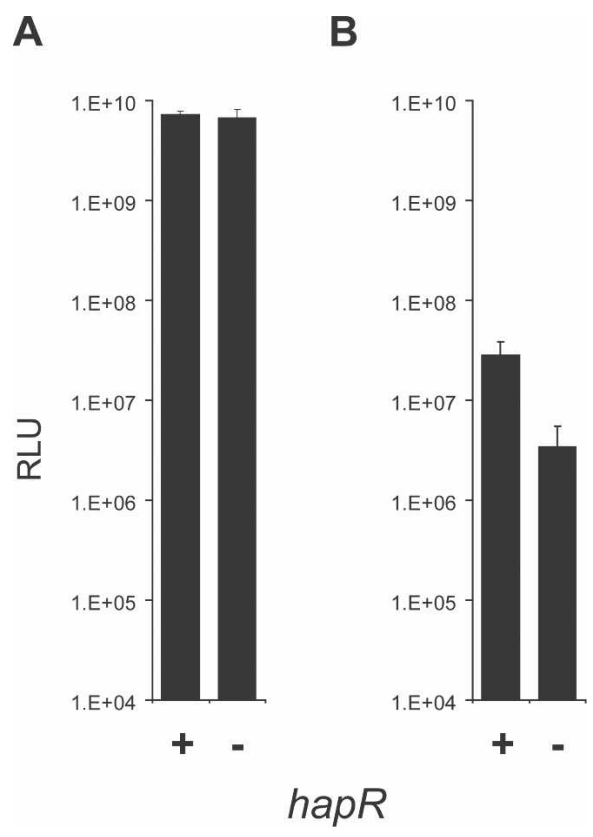

Figure 6. The HapR feedback loop is not relevant at steadystate high and low cell density conditions. Light production from the qrr4-lux promoter fusion was measured under steadystate conditions. (A) Wild-type $(+)$ and $\Delta$ hapR $(-)$ cells at steadystate low cell density $\left(\mathrm{OD}_{600}=0.03\right)$. (B) Wild-type $(+)$ and $\Delta$ hapR (-) cells at steady-state high cell density $\left(\mathrm{OD}_{600}=4\right)$. The experiment was repeated three times, each time in triplicate; error bars indicate one standard deviation from the mean. (RLU) Relative light units.

loop has little or no consequence on quorum sensing at the steady-state high cell density condition where HapR is abundant but LuxO-P levels are low, nor at the steadystate low cell density condition where LuxO-P is abundant but HapR levels are low.

\section{HapR and LuxO-P coexist at the transitions between low and high cell density mode}

Because coexistence of HapR and LuxO-P is required for the feedback loop to be operational, we hypothesize that the HapR feedback loop could be important for influencing the dynamics of the transitions into or out of the low and high cell density modes (N. Wingreen, pers. comm.). Consistent with this notion, autogenous regulation has previously been shown to affect the temporal responsiveness of bacterial transcriptional networks (Rosenfeld et al. 2002; Maeda and Sano 2006). We consider two scenarios here: the transition into high cell density mode and the transition out of high cell density mode.

In the first case, during the transition from the low to high cell density mode, AI levels increase, and the cells switch from the LuxO-P-dominated low cell density mode to the HapR-dominated high cell density mode. During this switch, LuxO-P and HapR could transiently coexist, allowing HapR to feedback-activate the qrr promoters. The resulting increase in Qrr levels, via their 
repression of hapR mRNA, could function to delay entry into high cell density mode.

In the second case, immediately following the switch from high to low cell density conditions, LuxO would become phosphorylated, and any pre-existing HapR could function to feedback-control the $q r r$ genes causing a dramatic increase in Qrr levels. This instantaneous increase in Qrr levels could, in turn, enable V. cholerae to accelerate the changes in gene expression required for entrance into individual cell mode.

\section{Direct HapR transcriptional autorepression controls the transition into high cell density mode.}

Figure 7A shows our examination of the first transition, from low to high cell density. Expression of the qrr genes decreases during this transition, and qrr4-lux levels decline more rapidly (i.e., at lower cell densities) in the absence of HapR ( $\Delta$ hapR, Fig. 7A, open squares) than in its presence (wild type, Fig. 7A, closed squares), consistent with the hypothesis that HapR feeds back to enhance qrr gene expression. To determine if the prolonged expression of the qrr genes during $V$. cholerae's transition into low cell density mode translates into delayed accumulation of HapR protein, we monitored HapR protein using a translational fusion of GFP to the first 10 amino acids of HapR and single-cell flow cytometry (Fig. 7B) in the presence (wild type, Fig. 7B, closed squares) or absence ( $\Delta$ hapR, Fig. 7B, open squares) of HapR autorepression. We verified that translation of this hapR-gfp construct is repressed by the Qrrs through sRNA-mRNA pairing (data not shown). At low cell density, HapR-GFP levels in the wild-type and $\Delta h a p R$ strains are indistinguishable. This is expected because the HapR-mediated feedback does not affect qrr expression under this con- dition (Fig. 6A), and direct hapR autorepression is also not effective at low cell density (Lin et al. 2005). However, as $V$. cholerae transitions into high cell density mode, Figure $7 \mathrm{~B}$ shows that more HapR-GFP accumulates in the $\Delta$ hapR strain than in the wild type. This result suggests that HapR feeds back to repress its own expression during the transition into high cell density mode. HapR-dependent repression could be a consequence of the prolonged qrr expression observed in Figure 7A, or alternatively, this could be due to HapR binding the hapR promoter and repressing its own transcription (Fig. 1; Lin et al. 2005). To distinguish between the two mechanisms, we deleted the HapR-binding site upstream of the hapR gene, thereby preventing direct hapR autorepression without altering Qrr repression of hapR translation. The resulting HapR-GFP levels are identical in the presence (wild type, Fig. 7C, closed squares) and absence ( $\Delta$ hapR, Fig. 7C, open squares) of the HapR feedback loop (Fig. 7C). Thus, the dynamics of the transition into quorum-sensing mode are affected by direct hapR autorepression, but not by HapR feedback on qrr transcription.

\section{The sRNA-mediated HapR feedback loop accelerates the transition out of high cell density mode}

As described above the HapR feedback loop on the qrr genes could be critical immediately following the switch from high to low cell density conditions, when LuxO-P and HapR are both abundant. To investigate this possibility, we simulated the switch from high to low cell density in $V$. cholerae cells containing and lacking the HapR feedback loop. Specifically, we pelleted high cell density wild-type and $\Delta$ hap $R$. cholerae cells, and immediately resuspended them in either cell-free spent-
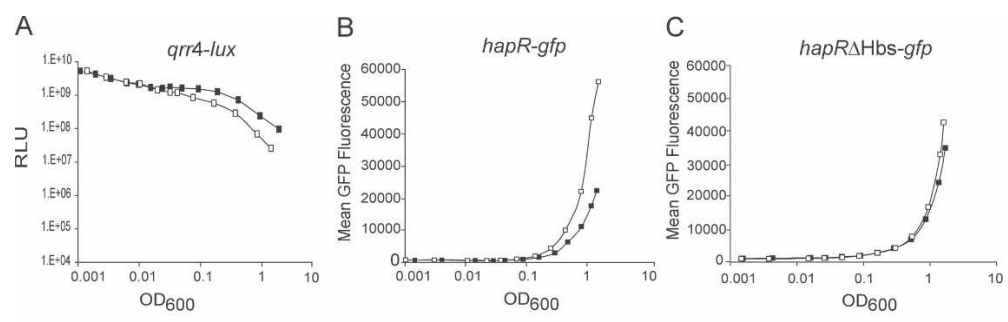

D

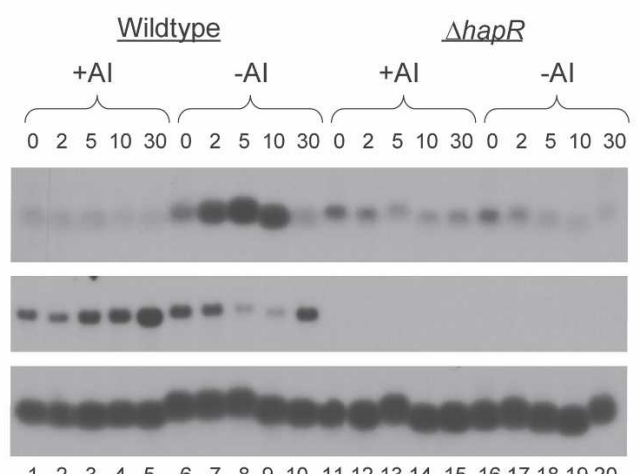

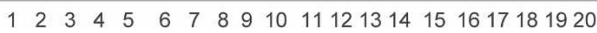

Qrr4

hapR

5S RNA

Lane
Figure 7. The HapR feedback accelerates the transition out of but not into high cell density mode. $(A)$ Light production from the qrr4-lux promoter fusion was measured during the transition from low to high cell population density with (closed squares) or without (open squares) HapR feedback. (B) HapR production (GFP fluorescence, mean value of 10,000 cells) during the transition into high cell density mode in cells with (wild type, closed squares) or without ( $\Delta$ hapR, open squares) HapR feedback. (C) HapR production (GFP fluorescence, mean value of 10,000 cells) during the transition into high cell density mode in the absence of direct hapR autorepression. The hapR-gfp construct lacking the HapRbinding site is denoted hapR $\Delta \mathrm{Hbs}-g f p$. Symbols are as described for $B .(D)$ High cell density cultures of wild-type (lanes 1-10) or $\Delta$ hapR (lanes 11-20) V. cholerae cells were pelleted, washed, and resuspended in either their own cell-free spent-culture fluids (+AI, lanes 1-5,11-15) or fresh medium (-AI, lanes 6-10,16-20). Aliquots were collected at the times indicated after wash, and the RNA species indicated on the right were visualized by Northern blotting. 5S RNA is shown as the loading control. The experiments were repeated twice. 
culture fluid (+AI, to simulate high cell density) or in fresh medium (-AI, to simulate low cell density). Subsequently, we monitored Qrr levels. As expected, no accumulation of Qrr sRNAs occurred in cells resuspended in the cell-free spent-culture fluid containing AIs (Fig. 7D, lanes 1-5, +AI). In contrast, when the wild-type cells were resuspended in fresh medium lacking AIs, Qrr levels increased within the first few minutes (Fig. 7D, lanes 6-8). By 30 min, the high cell density AI concentrations had been re-established, and Qrr levels once again began to decline (Fig. 7D, lanes 9-10). Consistent with these results, hapR mRNA levels continued to increase when the cells were reintroduced into medium containing AIs; however, following introduction into medium lacking AIs, a dramatic reduction in hapR mRNA occurred within $5 \mathrm{~min}$. Importantly, in the case of the $V$. cholerae $\Delta$ hapR mutant that does not possess the HapR feedback loop, no detectable alterations in Qrr levels occurred following either treatment (Fig. 7D, lanes 11-20). The loss of the rapid Qrr accumulation in AI-free medium indicates that, without the HapR feedback loop on qrr expression, LuxO-P-stimulation of the qrr promoters alone is not sufficient to mediate the entry into individual cell mode immediately following $V$. cholerae transitioning to low cell density. Thus, we conclude that the HapR-qrr feedback loop is critical for enabling a rapid switch from high to low cell density mode.

\section{Discussion}

Quorum sensing enables bacteria to transition between two distinct modes of gene expression: one appropriate for individual behaviors under low cell density conditions, and the other appropriate for community behaviors under high cell density conditions. In the wild, the transition from low to high cell density conditions presumably occurs gradually, as the cells multiply and AIs accumulate proportionally over time. In contrast, the transition from high to low cell population density could occur instantaneously; for example, following disruption of a biofilm (Zhu and Mekalanos 2003), or excretion from a host. It is likely, therefore, that quorum-sensing bacteria have evolved mechanisms to progressively respond to AI buildup, and to rapidly respond to the abrupt change to AI-free conditions. Indeed, regarding the low to high cell density transition, previous work shows that the topology of the LuxPQ-AI-2 receptor physically prevents it from clustering (Neiditch et al. 2006), suggesting that amplification of the quorum-sensing signal does not occur. This is in contrast to other ligand-receptor interactions (e.g., chemotaxis) in which small changes in ligand concentrations are amplified by the formation of cooperative signaling arrays of the cognate receptors (Maddock and Shapiro 1993; Parkinson et al. 2005).

Regarding the opposite transition, high to low cell population density, we provide evidence here supporting the notion that this transition is rapid. In V. cholerae, we find that the transition out of social mode and into individual cell mode is accelerated dramatically by a negative feedback loop. Specifically, upon removal of AIs, the master quorum-sensing regulator, $\mathrm{HapR}$, boosts production of the four individual cell mode-promoting sRNAs Qrr1-4, which, in turn, leads to degradation of hapR mRNA, all within minutes of the transition. This feedback loop requires functional HapR protein, but does not require base-pairing between hapR mRNA and Qrrl-4. Feedback occurs at the level of qrr transcription and appears to be indirect, but specific to the qrr promoters, rather than a general effect on LuxO-P- or $\sigma^{54}$-regulated promoters.

sRNA-mediated feedback on HapR functions independently of the direct transcriptional autorepression of hapR described previously (Chatterjee et al. 1996; Lin et al. 2005). Specifically, the sRNA-mediated feedback loop functions at the high to low cell density transition, while autorepression affects the transition from low to high cell density mode. This latter loop controls the steadystate level of hapR at high cell population density (Lin et al. 2005). In general, direct autorepression by transcriptional regulators accelerates the dynamics of the transition from low to high concentrations of active regulator, because autorepression provides a means to combine a strong promoter with controlled steady-state levels of the gene product (Rosenfeld et al. 2002; Camas et al. 2006). Autorepression is beneficial because accumulation of a regulator to levels beyond its functional range is undesirable due to the metabolic cost of unnecessary production of the regulator, possible toxic effects of an overabundance of the regulator, and the loss of sensitivity to external stimuli that function to terminate the regulator's activity. We show that, in the absence of autorepression, HapR levels increase more rapidly, and stabilize at higher steady-state levels than they do in the presence of autorepression (Fig. 7B-C). Thus, hapR autorepression, and not HapR feedback on the qrr genes, exclusively controls the transition into high cell density mode and allows a definitive response to AIs while avoiding hapR overproduction at the steady state.

Transcriptional autorepression accelerates the increase, but does not generally affect termination of the activity of a particular regulator because autorepression does not affect the degradation rate of the encoded gene product (Rosenfeld et al. 2002). Usually, genetic circuits employ an alternative strategy for rapid termination of a process driven by a particular transcriptional regulator. For example, in the case of the CI repressor that prevents prophage $\lambda$ induction, termination is accomplished by induced self-cleavage of the CI regulator protein (Roberts et al. 1978; Little 1984). In many metabolic networks, such as that controlling lactose metabolism in E. coli, the regulator (e.g., LacI) is rapidly inactivated by binding of an inducer molecule (e.g., allolactose) (Jacob and Monod 1961). In the context of quorum sensing, our results demonstrate that rapid termination of HapR activity is accomplished by a sRNA-based negative feedback loop. First, the Qrr sRNAs shorten the half-life of the hapR mRNA >30-fold (Lenz et al. 2004). Second, the feedback loop is particularly effective because synthesis of sRNAs is fast compared with protein synthesis. Third, because feedback occurs on multiple (four) qrr promot- 
ers, together, the four sRNAs contribute additively to the net increase in their concentration. Assuming each qrr promoter is fully activated (i.e., neither HapR nor LuxO-P levels is limiting), immediately following the transition to low cell density conditions, the total Qrr level increases four times faster than it would if there were only a single qrr gene subject to feedback control. Clearly, however, HapR inactivation at the level of protein activity or stability would be even more rapid than sRNA-based repression of hapR mRNA translation.

We consider several possibilities for why $V$. cholerae's transition out of social mode employs sRNA translational regulation of hapR. First, the metabolic cost for sRNA-based regulation is lower than that for protein degradation, because sRNAs act at an upstream step, thereby negating protein synthesis altogether. Second, recent work in our laboratory shows that the Qrr sRNAs act at targets other than hapR mRNA (Hammer and Bassler 2007), and, in at least one case, the Qrr sRNAs act as translational activators. Thus, the surge in Qrr sRNA production that follows immediately after the transition to low cell density conditions leads not only to rapid elimination of hapR, but also, perhaps more critically, could lead to the prompt production of one or more proteins required for transition into the individual cell mode (N. Wingreen, pers. comm.). Finally, in addition to the HapR-qrr feedback loop, it is possible that an as-yet-undescribed mechanism exists for inactivating HapR protein at low cell population density. Other members of the TetR family of transcriptional regulators are inactivated through the binding of particular small molecules (Beck et al. 1982). Hypothetically, a ligand could exist for HapR that would inactivate existing protein following the switch to low cell population density (De Silva et al. 2007).

Transcriptional activation of redundant sRNA genes by the product of their target mRNA has been documented in other bacterial systems (Reimmann et al. 2005; Kay et al. 2006). In Pseudomonas fluorescens CHA0, for example, three redundant small RNAs, RsmXYZ, sequester the proteins RsmA and RsmE that otherwise repress synthesis of exoproducts, such as antibiotics (Valverde et al. 2003; Kay et al. 2005; Reimmann et al. 2005). RsmA and RsmE, in turn, feedback activate transcription of $r s m X Y Z$ as well as enhance RsmXYZ stability (Reimmann et al. 2005). The mechanisms of these feedback circuits and the consequences on the biological processes controlled by the regulatory networks have not yet been defined.

It is well established that the success of bacterial pathogens often depends on rapid adjustment of gene expression to changing environmental conditions inside and outside of the host (Coote 2001; Beier and Gross 2006; Groisman and Mouslim 2006). This characteristic is clearly demonstrated in Salmonella typhimurium, where positive feedback by the "master" two-component virulence regulators $\mathrm{PhoP} / \mathrm{PhoQ}$ causes a surge in phoP transcription immediately following the switch to PhoP/PhoQ-activating conditions. The surge in phoP transcription is an absolute requirement for initiation of the S. typhimurium virulence cascade, and S. typhimurium cells lacking the feedback loop are avirulent (Shin et al. 2006). The infectious cycle of $V$. cholerae involves multiple transitions into and out of social mode. For example, $V$. cholerae cells in a high cell density biofilm structure are more likely to survive passage through the acidic gastric environment of a new host than are individual cells, but, subsequent to reaching the intestine, quorum-sensing-activated dispersal of the biofilm is required for individual cells to effectively colonize the intestinal epithelium and initiate virulence factor production (Zhu and Mekalanos 2003; Liu et al. 2007). This pattern repeats later in the infection when the cell population density has increased in the intestine, and quorum-sensing signals promote dispersal of individual cells. These cells are in the correct quorum-sensing mode (i.e., individual cell mode) to reinitiate colonization of additional regions of the intestine ( $\mathrm{Zhu}$ and Mekalanos 2003).

Together, this and previous work (Lin et al. 2005) show that the $V$. cholerae quorum-sensing circuit contains two negative feedback loops that enable the circuit to respond rapidly to changing AI levels. This network architecture is in stark contrast to the well-described LuxI/ LuxR quorum-sensing circuit of the related bacterium Vibrio fischeri. In $V$. fischeri, the LuxR transcription factor binds to AI produced by LuxI. AI-bound LuxR induces expression of bioluminescence genes and of the luxI gene (Engebrecht et al. 1983; Engebrecht and Silverman 1984). Thus, when an individual $V$. fischeri cell commits to the social mode because its LuxR is bound to signal, the LuxR-AI complex promotes the synthesis of additional AI. A positive feedback loop is established that floods the surrounding area with additional AI. This feedback loop forces neighboring cells to switch into the social mode. Thus, the $V$. fischeri regulatory circuitry appears configured to ensure a synchronous population-wide commitment to the social mode, which is critical to secure coordinated bioluminescence expression inside $V$. fischeri's squid host. In contrast, the quorum-sensing circuit of $V$. cholerae appears poised to enable individual cells to rapidly switch from the high to the low cell density mode. Both biofilm formation and virulence factor expression, two fundamental processes controlled by quorum sensing in $V$. cholerae, are activated in the low cell density state. Consistent with this, the two negative feedback loops in the $V$. cholerae quorum-sensing circuit ensure an immediate ability to produce biofilms and express virulence factors following a change in cell population density.

Multiple feedback loops are crucial elements of dynamic processes in biology such as in the cell cycle of Xenopus laevis (Pomerening et al. 2005), transient DNA competence in Bacillus subtilis (Suel et al. 2006), and induction of galactose uptake in Saccharomyces cerevisiae (Ramsey et al. 2006). Here we show that HapR, the master regulator of quorum sensing in $V$. cholerae, is autoregulated by two negative feedback loops. What is the benefit of having two negative feedback loops acting on the same gene product? We demonstrate that the two 
feedback loops contribute to distinct characteristics of the system. The direct transcriptional feedback loop promotes a strong response to AIs during the transition into social mode, while preventing runaway expression of hapR at high cell density. The sRNA-mediated feedback loop, on the other hand, tremendously accelerates the exit from social mode.

\section{Materials and methods}

\section{Bacterial strains and culture conditions}

$V$. cholerae strains used in this study are derivatives of El Tor strain C6706str2 (Thelin and Taylor 1996). E. coli strains S17$1 \lambda$ ir (de Lorenzo and Timmis 1994), ElectroMAX DH10B (Invitrogen), and One Shot (Invitrogen) were used for cloning. All strains were grown in LB broth with aeration or on LB agar at $30^{\circ} \mathrm{C}$. Antibiotics were used at the following concentrations: $200 \mu \mathrm{g} \mathrm{mL}{ }^{-1}$ ampicillin, $100 \mu \mathrm{g} \mathrm{mL}{ }^{-1}$ kanamycin, $10 \mu \mathrm{gL}^{-1}$ chloramphenicol, $50 \mu \mathrm{g} \mathrm{mL}^{-1}$ polymyxin $\mathrm{B}, 1000 \mu \mathrm{mL}^{-1}$ streptomycin, and $10 \mu \mathrm{g} \mathrm{mL}^{-1}$ tetracycline.

\section{DNA manipulations}

All V. cholerae strains and plasmids used in this study are listed in Supplemental Table S1. DNA manipulations were performed according to Sambrook et al. (1989) unless otherwise noted. Herculase polymerase (Stratagene) was used for PCR reactions in cloning procedures, whereas Taq polymerase (Roche) was used for all other PCR reactions. dNTPs, restriction endonucleases, and T4 ligase were obtained from New England Biolabs. DNA purification kits were obtained from Qiagen. V. cholerae inframe deletions were constructed by the method of Skorupski and Taylor (1996). The lux transcriptional fusion plasmids were constructed as in Lenz et al. (2004) and were introduced into $V$. cholerae by conjugation. Primer sequences are available on request. To construct the Pqrr ${ }^{\text {scr-}}$-lux promoter construct, overlapping single-stranded oligonucleotides were designed containing the 90 bases to be incorporated in random order, flanked by the qrr4 promoter start and end regions. PCR was performed on the oligonucleotides using BamHI/SpeI-tailed primers, and the PCR product was cloned into pBBRlux. IPTG-inducible hapR expression constructs were cloned into the StuI/BamHI sites of pFED342, a modified version of pEVS143 (Dunn et al. 2006). pSLS13 contains the full-length hapR gene starting at the +1 transcriptional start site (Lin et al. 2005). pSLS28 contains the hapR ORF (starting at +80 ) linked to the RBS provided by pFED342. pSLS30 contains full-length hapR with the R18C missense mutation cloned from BH267 (Hammer and Bassler 2003). The hapR-gfp protein fusion was constructed by three-step PCR mutagenesis. Briefly, the hapR promoter including the first 10 codons of the hapR ORF and the $g f p$ ORF from $\mathrm{pCMW} 1$ (Waters and Bassler 2006) were PCR-amplified independently. The two resulting PCR products were combined and served as a template for a third PCR reaction using hapR upstream and gfp downstream primers. The resulting hapR-gfp fusion product was cloned into pCR-blunt II-TOPO (Invitrogen) to generate pSLS71. The PstI/BamHI fragment of pSLS71 was cloned into the PstI/ BamHI sites of cosmid pLAFR2 to generate pSLS73. The HapRbinding site of the hapR-gfp promoter (bases +12 to +31 ) was deleted by Quickchange mutagenesis (Invitrogen) of pSLS71 to generate pSLS74. The PstI/BamHI fragment of pSLS74 was cloned into pLAFR2 to make pSLS75.

\section{Bioluminescence assays}

Bioluminescence was measured as described previously (Lenz et al. 2004). For time-course experiments, overnight cultures without IPTG were diluted such that each culture was at the identical cell density ( 1:1000 dilution). The cultures were grown in $25 \mathrm{~mL}$ of LB broth containing $50 \mu \mathrm{M} \mathrm{IPTG}$ and the appropriate antibiotics (except in the experiment shown in Fig. 7A, in which each culture was diluted 1:50,000,000 and grown without IPTG). Light production and $\mathrm{OD}_{600}$ were measured every $\sim 45$ min. For the single-time-point experiments shown in Figure 5C, overnight cultures without IPTG were diluted 1:1000 and grown with $50 \mu \mathrm{M}$ IPTG to $\mathrm{OD}_{600}=0.1$, at which point light production was measured. In Figure 6, overnight cultures were diluted 1:50,000,000 and light production was measured at $\mathrm{OD}_{600}=0.03$ (Fig. 6A) and $\mathrm{OD}_{600}=4$ (Fig. 6B). Relative light units $(\mathrm{RLU})$ are defined as counts per minute per milliliter per $\mathrm{OD}_{600}$.

\section{Northern blot analysis}

Cultures used for RNA preparations were grown to an $\mathrm{OD}_{600}$ of 0.10 (single-time-point Northern blots). For time courses, overnight cultures of wild-type $V$. cholerae were diluted 1:1000 and aliquots for RNA preparations were collected approximately every hour. RNA was extracted with Trizol (Invitrogen) and chloroform. RNA was precipitated with isopropanol, washed with $75 \%$ ethanol, and resuspended in DEPC water. Northern blots were performed as described (Martin et al. 1989), except ssDNA probes were synthesized using the Strip-EZ PCR Probe Synthesis and Removal Kit (Ambion). Probe primers are available on request. In some cases, membranes were stripped and reprobed for an RNA species of a different size, but Qrr1-4 were always probed on fresh unstripped membranes.

\section{EMSAs}

HapR was purified with the IMPACT protein purification system (New England Biolabs) using the expression plasmid pTYB11 and the protocol described in the manufacturer's instructions. Purified HapR was stored in $20 \mathrm{mM}$ Tris (pH 7.5), 1 mM EDTA, $10 \mathrm{mM} \mathrm{NaCl}$, and $0.1 \mathrm{mM}$ DTT with $20 \%$ glycerol as described previously (Lin et al. 2005). DNA probes for gel mobility shift analyses were generated using $5{ }^{\prime}$-tagged fluorescent primers in a standard PCR reaction and were purified using the Zymoclean Gel DNA Recovery Kit (Zymo Research). The $a p h A$ nonshift and shift control probes were constructed to contain the region from 691 to $371 \mathrm{nt}$ upstream of the aphA translation start site and from $327 \mathrm{nt}$ upstream of to $22 \mathrm{nt}$ downstream from the aphA translation start site, respectively. The qrr promoter probes contained $500 \mathrm{nt}$ upstream of the respective transcription start sites. Each probe $(10 \mathrm{nM})$ was incubated with the indicated amount of $\operatorname{HapR}(0,50,250$, and $500 \mathrm{nM})$ and $1 \mathrm{~mL}$ of $1 \mathrm{mg} / \mathrm{mL}$ poly-dIdC in a final volume of $20 \mathrm{~mL}$ for $15 \mathrm{~min}$ at $30^{\circ} \mathrm{C}$. Gel mobility shifts were performed on a $5 \%$ TAE-polyacrylimide gel and were visualized using a Storm 860 Imaging System (Molecular Dynamics).

\section{Single-cell fluorescence analysis}

Overnight cultures of wild type/pSLS73, $\Delta$ hapR/pSLS73, wild type/pSLS75, and $\Delta$ hapR/pSLS75 were diluted 1:50,000,000 and grown to $\mathrm{OD}_{600} \sim 0.001$ prior to initiating measurements. Aliquots were analyzed every $30 \mathrm{~min}$ for $6 \mathrm{~h}$. The fluorescence of individual cells was measured on a Becton Dickinson FACS Aria cell sorter. Data were analyzed using Becton Dickinson FACSDiva software. 


\section{Acknowledgments}

We thank Ned Wingreen, Sandeep Krishna, Thomas Silhavy, Michael A. Fisher, and members of the Bassler laboratory for helpful ideas and suggestions. This work was supported by HHMI, NIH grant 5R01GM065859, and NSF grant MCB0343821.

\section{References}

Beck, C.F., Mutzel, R., Barbe, J., and Muller, W. 1982. A multifunctional gene (tetR) controls Tn10-encoded tetracycline resistance. J. Bacteriol. 150: 633-642.

Beier, D. and Gross, R. 2006. Regulation of bacterial virulence by two-component systems. Curr. Opin. Microbiol. 9: 143152.

Buck, M., Gallegos, M.T., Studholme, D.J., Guo, Y., and Gralla, J.D. 2000. The bacterial enhancer-dependent $\sigma^{54}\left(\sigma^{\mathrm{N}}\right)$ transcription factor. J. Bacteriol. 182: 4129-4136.

Camas, F.M., Blazquez, J., and Poyatos, J.F. 2006. Autogenous and nonautogenous control of response in a genetic network. Proc. Natl. Acad. Sci. 103: 12718-12723.

Chatterjee, J., Miyamoto, C.M., and Meighen, E.A. 1996. Autoregulation of luxR: The Vibrio harveyi lux-operon activator functions as a repressor. Mol. Microbiol. 20: 415-425.

Coote, J.G. 2001. Environmental sensing mechanisms in Bordetella. Adv. Microb. Physiol. 44: 141-181.

de Lorenzo, V. and Timmis, K.N. 1994. Analysis and construction of stable phenotypes in Gram-negative bacteria with Tn5- and Tn10-derived minitransposons. Methods Enzymol. 235: 386-405.

De Silva, R.S., Kovacikova, G., Lin, W., Taylor, R.K., Skorupski, K., and Kull, F.J. 2007. Crystal structure of the Vibrio cholerae quorum sensing regulatory protein HapR. J. Bacteriol. 189: 5683-5691.

Dunn, A.K., Millikan, D.S., Adin, D.M., Bose, J.L., and Stabb, E.V. 2006. New rfp- and pES213-derived tools for analyzing symbiotic Vibrio fischeri reveal patterns of infection and lux expression in situ. Appl. Environ. Microbiol. 72: 802-810.

Engebrecht, J. and Silverman, M. 1984. Identification of genes and gene products necessary for bacterial bioluminescence. Proc. Natl. Acad. Sci. 81: 4154-4158.

Engebrecht, J., Nealson, K., and Silverman, M. 1983. Bacterial bioluminescence: Isolation and genetic analysis of functions from Vibrio fischeri. Cell 32: 773-781.

Freeman, J.A. and Bassler, B.L. 1999. A genetic analysis of the function of LuxO, a two-component response regulator involved in quorum sensing in Vibrio harveyi. Mol. Microbiol. 31: 665-677.

Groisman, E.A. and Mouslim, C. 2006. Sensing by bacterial regulatory systems in host and non-host environments. Nat. Rev. Microbiol. 4: 705-709.

Hammer, B.K. and Bassler, B.L. 2003. Quorum sensing controls biofilm formation in Vibrio cholerae. Mol. Microbiol. 50: 101-104.

Hammer, B.K. and Bassler, B.L. 2007. Regulatory small RNAs circumvent the conventional quorum sensing pathway in pandemic Vibrio cholerae. Proc. Natl. Acad. Sci. 104: 11145-11149.

Henke, J.M. and Bassler, B.L. 2004. Bacterial social engagements. Trends Cell Biol. 14: 648-656.

Huo, Y.X., Nan, B.Y., You, C.H., Tian, Z.X., Kolb, A., and Wang, Y.P. 2006. FIS activates glnAp2 in Escherichia coli: Role of a DNA bend centered at -55 , upstream of the transcription start site. FEMS Microbiol. Lett. 257: 99-105.

Jacob, F. and Monod, J. 1961. Genetic regulatory mechanisms in the synthesis of proteins. J. Mol. Biol. 3: 318-356.

Jobling, M.G. and Holmes, R.K. 1997. Characterization of hapR, a positive regulator of the Vibrio cholerae HA/protease gene hap, and its identification as a functional homologue of the Vibrio harveyi luxR gene. Mol. Microbiol. 26: 1023-1034.

Kay, E., Dubuis, C., and Haas, D. 2005. Three small RNAs jointly ensure secondary metabolism and biocontrol in Pseudomonas fluorescens CHA0. Proc. Natl. Acad. Sci. 102: 17136-17141.

Kay, E., Humair, B., Denervaud, V., Riedel, K., Spahr, S., Eberl, L., Valverde, C., and Haas, D. 2006. Two GacA-dependent small RNAs modulate the quorum-sensing response in Pseudomonas aeruginosa. J. Bacteriol. 188: 6026-6033.

Kovacikova, G. and Skorupski, K. 2002. Regulation of virulence gene expression in Vibrio cholerae by quorum sensing: HapR functions at the aphA promoter. Mol. Microbiol. 46: 11351147.

Krishnan, H.H., Ghosh, A., Paul, K., and Chowdhury, R. 2004. Effect of anaerobiosis on expression of virulence factors in Vibrio cholerae. Infect. Immun. 72: 3961-3967.

Lenz, D.H. and Bassler, B.L. 2007. The small nucleoid protein Fis is involved in Vibrio cholerae quorum sensing. Mol. Microbiol. 63: 859-871.

Lenz, D.H., Mok, K.C., Lilley, B.N., Kulkarni, R.V., Wingreen, N.S., and Bassler, B.L. 2004. The small RNA chaperone Hfq and multiple small RNAs control quorum sensing in Vibrio harveyi and Vibrio cholerae. Cell 118: 69-82.

Lenz, D.H., Miller, M.B., Zhu, J., Kulkarni, R.V., and Bassler, B.L. 2005. CsrA and three redundant small RNAs regulate quorum sensing in Vibrio cholerae. Mol. Microbiol. 58: 1186-1202.

Lin, W., Kovacikova, G., and Skorupski, K. 2005. Requirements for Vibrio cholerae HapR binding and transcriptional repression at the hapR promoter are distinct from those at the aphA promoter. J. Bacteriol. 187: 3013-3019.

Little, J.W. 1984. Autodigestion of lexA and phage $\lambda$ repressors. Proc. Natl. Acad. Sci. 81: 1375-1379.

Liu, Z., Stirling, F.R., and Zhu, J. 2007. Temporal quorum-sensing induction regulates Vibrio cholerae biofilm architecture. Infect. Immun. 75: 122-126.

Maddock, J.R. and Shapiro, L. 1993. Polar location of the chemoreceptor complex in the Escherichia coli cell. Science 259: 1717-1723.

Maeda, Y.T. and Sano, M. 2006. Regulatory dynamics of synthetic gene networks with positive feedback. J. Mol. Biol. 359: $1107-1124$.

Martin, M., Showalter, R., and Silverman, M. 1989. Identification of a locus controlling expression of luminescence genes in Vibrio harveyi. J. Bacteriol. 171: 2406-2414.

Masse, E., Escorcia, F.E., and Gottesman, S. 2003. Coupled degradation of a small regulatory RNA and its mRNA targets in Escherichia coli. Genes \& Dev. 17: 2374-2383.

Miller, M.B., Skorupski, K., Lenz, D.H., Taylor, R.K., and Bassler, B.L. 2002. Parallel quorum sensing systems converge to regulate virulence in Vibrio cholerae. Cell 110: 303 314.

Miller, S.T., Xavier, K.B., Campagna, S.R., Taga, M.E., Semmelhack, M.F., Bassler, B.L., and Hughson, F.M. 2004. Salmonella typhimurium recognizes a chemically distinct form of the bacterial quorum-sensing signal AI-2. Mol. Cell 15: 677687.

Miyamoto, C.M., Smith, E.E., Swartzman, E., Cao, J.G., Graham, A.F., and Meighen, E.A. 1994. Proximal and distal sites bind LuxR independently and activate expression of the Vibrio harveyi lux operon. Mol. Microbiol. 14: 255-262.

Morett, E. and Buck, M. 1989. In vivo studies on the interaction 
of RNA polymerase- $\sigma^{54}$ with the Klebsiella pneumoniae and Rhizobium meliloti nifH promoters. The role of NifA in the formation of an open promoter complex. J. Mol. Biol. 210: 65-77.

Neiditch, M.B., Federle, M.J., Pompeani, A.J., Kelly, R.C., Swem, D.L., Jeffrey, P.D., Bassler, B.L., and Hughson, F.M. 2006. Ligand-induced asymmetry in histidine sensor kinase complex regulates quorum sensing. Cell 126: 1095-1108.

Nye, M.B., Pfau, J.D., Skorupski, K., and Taylor, R.K. 2000. Vibrio cholerae $\mathrm{H}-\mathrm{NS}$ silences virulence gene expression at multiple steps in the ToxR regulatory cascade. J. Bacteriol. 182: 4295-4303.

Pan, W. and Spratt, B.G. 1994. Regulation of the permeability of the gonococcal cell envelope by the mtr system. Mol. Microbiol. 11: 769-775.

Parkinson, J.S., Ames, P., and Studdert, C.A. 2005. Collaborative signaling by bacterial chemoreceptors. Curr. Opin. Microbiol. 8: 116-121.

Pomerening, J.R., Kim, S.Y., and Ferrell Jr., J.E. 2005. Systemslevel dissection of the cell-cycle oscillator: Bypassing positive feedback produces damped oscillations. Cell 122: 565 578.

Popham, D.L., Szeto, D., Keener, J., and Kustu, S. 1989. Function of a bacterial activator protein that binds to transcriptional enhancers. Science 243: 629-635.

Ramsey, S.A., Smith, J.J., Orrell, D., Marelli, M., Petersen, T.W., de Atauri, P., Bolouri, H., and Aitchison, J.D. 2006. Dual feedback loops in the GAL regulon suppress cellular heterogeneity in yeast. Nat. Genet. 38: 1082-1087.

Reimmann, C., Valverde, C., Kay, E., and Haas, D. 2005. Posttranscriptional repression of GacS/GacA-controlled genes by the RNA-binding protein RsmE acting together with RsmA in the biocontrol strain Pseudomonas fluorescens CHA0. J. Bacteriol. 187: 276-285.

Rimsky, S. 2004. Structure of the histone-like protein H-NS and its role in regulation and genome superstructure. Curr. Opin. Microbiol. 7: 109-114.

Roberts, J.W., Roberts, C.W., and Craig, N.L. 1978. Escherichia coli recA gene product inactivates phage $\lambda$ repressor. Proc. Natl. Acad. Sci. 75: 4714-4718.

Rosenfeld, N., Elowitz, M.B., and Alon, U. 2002. Negative autoregulation speeds the response times of transcription networks. J. Mol. Biol. 323: 785-793.

Sambrook, J., Fritsch, E.F., and Maniatis, T. 1989. Molecular cloning: A laboratory manual. Cold Spring Harbor Laboratory Press, Cold Spring Harbor, NY.

Schauder, S., Shokat, K., Surette, M.G., and Bassler, B.L. 2001. The LuxS family of bacterial autoinducers: Biosynthesis of a novel quorum-sensing signal molecule. Mol. Microbiol. 41: 463-476.

Shin, D., Lee, E.J., Huang, H., and Groisman, E.A. 2006. A positive feedback loop promotes transcription surge that jumpstarts Salmonella virulence circuit. Science 314: 1607-1609.

Skorupski, K. and Taylor, R.K. 1996. Positive selection vectors for allelic exchange. Gene 169: 47-52.

Smith, R.S. and Iglewski, B.H. 2003. P. aeruginosa quorumsensing systems and virulence. Curr. Opin. Microbiol. 6: 5660 .

Suel, G.M., Garcia-Ojalvo, J., Liberman, L.M., and Elowitz, M.B. 2006. An excitable gene regulatory circuit induces transient cellular differentiation. Nature 440: 545-550.

Swinger, K.K. and Rice, P.A. 2004. IHF and HU: Flexible architects of bent DNA. Curr. Opin. Struct. Biol. 14: 28-35.

Tendeng, C., Badaut, C., Krin, E., Gounon, P., Ngo, S., Danchin, A., Rimsky, S., and Bertin, P. 2000. Isolation and characterization of vicH, encoding a new pleiotropic regulator in $\mathrm{Vib}$ - rio cholerae. J. Bacteriol. 182: 2026-2032.

Thelin, K.H. and Taylor, R.K. 1996. Toxin-coregulated pilus, but not mannose-sensitive hemagglutinin, is required for colonization by Vibrio cholerae O1 El Tor biotype and O139 strains. Infect. Immun. 64: 2853-2856.

Valverde, C., Heeb, S., Keel, C., and Haas, D. 2003. RsmY, a small regulatory RNA, is required in concert with RsmZ for GacA-dependent expression of biocontrol traits in Pseudomonas fluorescens CHA0. Mol. Microbiol. 50: 1361-1379.

Waters, C.M. and Bassler, B.L. 2005. Quorum sensing: Cell-tocell communication in bacteria. Annu. Rev. Cell Dev. Biol. 21: $319-346$

Waters, C.M. and Bassler, B.L. 2006. The Vibrio harveyi quorum-sensing system uses shared regulatory components to discriminate between multiple autoinducers. Genes \& Dev. 20: $2754-2767$.

Wedel, A. and Kustu, S. 1995. The bacterial enhancer-binding protein NTRC is a molecular machine: ATP hydrolysis is coupled to transcriptional activation. Genes \& Dev. 9: 2042 2052.

Xavier, K.B. and Bassler, B.L. 2003. LuxS quorum sensing: More than just a numbers game. Curr. Opin. Microbiol. 6: 191197.

Zhu, J. and Mekalanos, J.J. 2003. Quorum sensing-dependent biofilms enhance colonization in Vibrio cholerae. Dev. Cell 5: 647-656. 


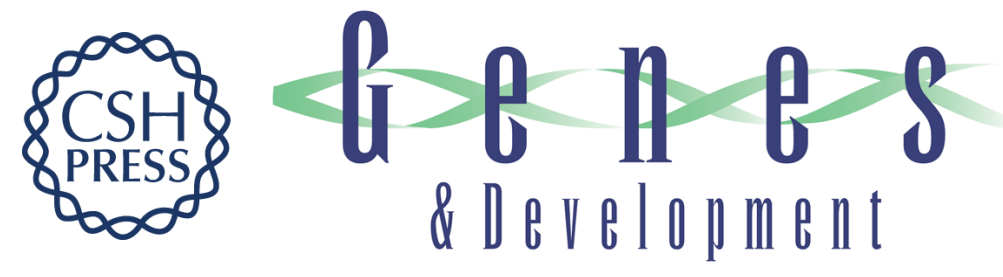

\section{A negative feedback loop involving small RNAs accelerates Vibrio cholerae 's transition out of quorum-sensing mode}

Sine L. Svenningsen, Christopher M. Waters and Bonnie L. Bassler

Genes Dev. 2008, 22:

Access the most recent version at doi:10.1101/gad.1629908

Supplemental
Material $\quad$ http://genesdev.cshlp.org/content/suppl/2008/01/16/22.2.226.DC1

References This article cites 62 articles, 25 of which can be accessed free at:

http://genesdev.cshlp.org/content/22/2/226.full.html\#ref-list-1

License

Email Alerting Receive free email alerts when new articles cite this article - sign up in the box at the top

Service right corner of the article or click here.

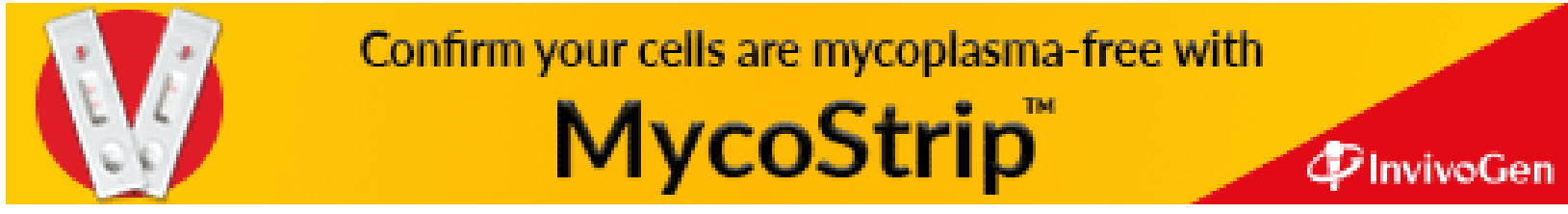

